\title{
Matériaux de construction
}

\section{Claude Simon}

\section{(2) OpenEdition}

\section{Journals}

Édition électronique

URL : https://journals.openedition.org/ccs/590

DOI : $10.4000 /$ ccs.590

ISSN : 2558-782X

\section{Éditeur :}

Presses universitaires de Rennes, Association des lecteurs de Claude Simon

\section{Édition imprimée}

Date de publication : 30 novembre 2008

Pagination : $9-17$

ISBN : 9782354120351

ISSN : 1774-9425

\section{Référence électronique}

Claude Simon, « Matériaux de construction », Cahiers Claude Simon [En ligne], 4 | 2008, mis en ligne le 21 septembre 2017, consulté le 26 avril 2022. URL : http://journals.openedition.org/ccs/590 ; DOI : https://doi.org/10.4000/ccs.590 


\section{MATÉRIAUX DE CONSTRUCTION*}

\section{Claude Simon}

Acre et entêtante odeur de vinaigre stagnant dans la maison : elle avait l'habitude de verser les fonds de bouteille dans deux pots de grès recouverts de morceaux d'un tulle grisâtre et parsemé de trous (ce qui obligeait à le mettre en double de façon à ce que toujours un trou corresponde à une partie intacte), tulle semblable à ces voiles de mariées qu'il n'avait jamais été.

L'enfant vagissant dans la voiture aux ressorts cassés la capote relevée un carré de cette même gaze décolorée et trouée également mise en double faisant tente à partir du haut de la capote pour le protéger des mouches.

On les sentait aussi dans la maison minuscules agaçantes plus moucherons que mouches points noirs aux ailes minuscules obsédants se posant disparaissant menu chatouillis miniatures d'insectes.

Mouches du vinaigre se reproduisant se multipliant à une vitesse qui permet paraît-il d'étudier sur elles les mutations de ces comment s'appellent (chromosomes, gènes ?) éléments dont les modifications déterminent l'évolution de l'espèce.

Plusieurs générations en quelques heures (ou jours) de sorte que c'est comme si à côté de notre temps se déroulait un autre temps filant à la vitesse accélérée comme un express doublant un train omnibus.

* Texte publié dans les Lettres nouvelles (directeur Maurice Nadeau) en décembre 1960 (pp. 112-122). Réédité avec l'aimable autorisation de Réa Simon. 
Me rappelant je ne sais pourquoi (sans doute à cause de ses travaux sur le vin la fermentation les levures) ce savant qu'avec agacement puis fureur je m'étais vu proposer en exemple pendant toute mon enfance, l'entendant encore me répéter sur ce ton sentencieux fervent et son visage douloureusement outragé comme pour devancer prévenir ricanements ou blasphème la phrase qu'il aurait un jour prononcée « Un peu de science éloigne de Dieu beaucoup en rapproche », revoyant aussi sa maison aujourd'hui transformée en musée (dans le bas de la ville) avec une plaque en marbre gravé sur le mur entièrement recouvert de vigne-vierge, mur de feuille vert foncé rougissant à l'automne, maison qui était à mes yeux comme un symbole d'austérité bienséante la dernière à gauche avant d'arriver au pont, on entendait le bruit continu de la rivière qui descendait la ville en une série de courtes cascades passant par les abattoirs les moulins des tailleurs de diamants, d'une couleur vert sombre et opaque se souillant au fur et à mesure exhalant une fade senteur de vase et d'égout, il n'en coulait plus à la fin (sous ce pont) qu'un filet d'eau stagnante souillée de détritus de légumes de papiers de boîtes de conserves vides à moitié submergées dérivant lentement sur la surface poussiéreuse sombre et malodorante entre les berges gluantes, et en face de la maison il y avait un garage avec un atelier de réparations la façade constellée de réclames aux vives couleurs pour des marques de lubrifiants ou de pneus, Veedol : jeune homme en chemise kaki et combinaison bleue à plastron et bretelles les manches retroussées sur des avant-bras musculeux la chevelure calamistrée (calaminée) tendant avec un sourire vainqueur vers le spectateur un bidon dans sa main qui a l'air de jaillir en avant de l'affiche, ou encore Hutchinson : rémouleur hirsute à la barbe hérissée coiffé d'un chapeau claque en accordéon aiguisant un couteau de cuisine sur un pneu, regardé par un petit chien (roquet) blanc à l'air étonné, les senteurs d'essence de cambouis et de dissolution se mêlant dans la chaleur d'août aux relents surs qui montaient de la rivière

la maison, le mur de feuilles, et celui qui avait habité là assis maintenant en effigie de bronze sur un fauteuil de bronze au centre du rond-point d'arbres qui terminait la promenade où se tenait tous les mois la foire aux bestiaux, figuraient sur les cartes postales pour touristes au même titre que le clocher de l'église et les tours des an- 
ciens remparts, cartes postales à prétentions artistiques tirées sur un papier sépia ou bleu avec un cache flou qui faisait apparaître la statue le clocher la cascade des abattoirs ou la vieille tour au centre d'un halo blanc comme il arrive que l'on voie les choses lorsqu'on cherche à les évoquer par le souvenir c'est-à-dire chacune ou un détail apparaissant avec une précision photographique mais isolé de son contexte par une sorte de brume ; je me rappelle que sur chacune des quatre faces du socle il y avait un bas-relief également en bronze vert-noir dont l'un représentait le fameux épisode du premier vaccin expérimenté sur le jeune berger mordu par un chien enragé (et dont l'histoire qui m'avait été plusieurs fois racontée enjolivée par une série de détails devenus légendaires au point d'en faire une sorte de saga exemplaire et un peu niaise qui se brouillait vaguement dans mon esprit (sans doute à cause de la lutte que, prétendait-on, le jeune pâtre avait livrée au chien contre lequel il s'était vaillamment défendu à coups de sabots) avec celle de la chèvre de Monsieur Seguin, le savant assis sur une chaise l'air soucieux et sévère coiffé d'une sorte de calotte cylindrique tourné vers l'enfant poussé vers lui comme pour un sacrifice par une religieuse à la grande cornette la main du vieux savant armée d'une seringue à la hauteur du bas ventre de l'enfant un groupe de messieurs barbus et graves massés derrière la chaise regardant la scène ou parlant entre eux sans doute à voix basse car leurs têtes sont penchées l'une vers l'autre comme des gens qui conversent de bouche à oreille.

Électricité allumée à toute heure de la journée dans son bureau ou du moins ce qu'on appelait ainsi les volets tirés en permanence ne laissant voir qu'un mince rai de l'éblouissante et torride lumière extérieure pourtant déjà atténuée par le grillage à moustiques rouillé et malgré cela si violente encore qu'il était presque impossible de fixer sans être ébloui cette fente où la lumière semblait bouillonner à la façon d'un acide c'est-à-dire s'immisçant de force mordant rongeant les bords presque joints des volets dont on ne recommençait à pouvoir distinguer la ligne nette que le soir lorsqu'elle s'apaisait un peu, seul moment (le crépuscule) où il se décidait à les pousser 
dans le crissement agaçant les dents des grains de cette poussière aussi sèche que le sable accumulée là par le vent et qui coincés entre le bas du ventail et la dalle de l'appui avaient fini par la rayer d'une multitude de cercles concentriques et blancs gravés dans la pierre grise par les minuscules parcelles de silex, mais même alors (en fait c'était presque à la nuit tombante) l'ampoule qui pendait nue sous l'abat-jour de tôle à la peinture (blanche en dessous, verte au-dessus) écaillée (et plus exactement non pas pendant - c'est-à-dire à la verticale sous le point où le fil se détachait du plafond (au milieu de celuici) mais tirée (c'est-à-dire le tout : l'ampoule, l'abat-jour, le fil engagé trois fois dans les poulies et le contrepoids de porcelaine blanche en forme d'œuf) à l'aide d'une ficelle sommairement nouée à un clou planté dans le mur au-dessus du bureau de façon à mieux l'éclairer), même alors donc l'ampoule restait allumée la seule différence étant que pendant un moment (celui qui séparait le crépuscule de la nuit) on pouvait distinguer sans être ébloui son filament incandescent et jaunâtre, non seulement je pense parce qu'alors la lumière pénétrant du dehors était plus forte mais parce qu'elle (l'ampoule) était vieille et fatiguée (et apparemment éternelle comme le donnait à croire l'accumulation des chiures de mouches qui témoignaient d'années d'usage) ; puis elle se remettait à briller, c'est-à-dire que passé cet étroit et équivoque laps de temps où les deux lumières (la naturelle et l'artificielle) semblaient autorisées à s'affronter (l'une - celle du jour - impétueuse éphémère mourant presque aussitôt comme si sa brusque irruption sa brusque intrusion dans ce monde défendu et sa brutale victoire avaient d'un seul coup épuisé toute sa force, l'autre constante, indifférente, reprenant peu à peu possession de son domaine), passé donc cette demi-heure (rarement plus) qui était semblait-il comme une concession faite à ce principe ou à cette coutume qui veut qu'il y ait dans une période de vingt-quatre heures un temps consacré au jour et un temps consacré aux ténèbres, elle éclairait de nouveau seule de sa lumière égale intemporelle et jaune l'étroite pièce tapissée d'un papier verdâtre à raies et presque tout entière occupée par un de ces bureaux sans style et non pas d'ébène mais de simple bois peint en noir aux pieds tournés et surmonté d'une étagère-classeur où s'empilaient ou plutôt se déversaient sur lui (formant une sorte de plan de clivage hérissé et confus) un fouillis de vieilles 
factures, de feuilles de paie, de lettres de négociants ou de courtiers en vins, de tables correctives pour mustimètres ou alcoomètres, lettres factures ou colonnes de chiffres uniformément maculées de cercles ou de demi-cercles baveux couleur lilas pâle et poisseux laissés par les socles des éprouvettes qu'il posait un peu partout à demi pleines de mistelle ou de résidus distillés, une odeur douceâtre et entêtante d'alcool à brûler de sucre et de moût chauffé flottant là elle aussi semblait-il en permanence même quand ce n'était pas l'époque des vendanges, me demandant par quel miracle il n'avait jamais mis le feu avec ce Dujardin-Salleron posé à même le bureau au milieu des papiers en désordre et sous lequel brûlaient à toute heure comme ces veilleuses ces pieux lumignons allumés dans les églises au pied des statues ou des images saintes la petite lampe avec sa mèche torsadée lovée comme un serpent dans l'alcool jaunâtre et dont la flamme veloutée léchait sans bruit la bouilloire de cuivre, oscillant paresseusement au moindre déplacement d'air par exemple quand on pénétrait dans la pièce pénétrant en même temps semblait-il (au sortir de l'éclatante étourdissante et même bruyante si l'on peut dire et même cacophonique lumière du dehors) dans un univers fixe où le temps ne s'écoulait pas à la même vitesse si tant est qu'il s'écoulât (puisque rien ou presque rien n'y distinguait le jour de la nuit) et où l'air jamais renouvelé conservait comme un parfum fondamental les subtiles et lourdes émanations de la décomposition en simples produits chimiques de ce que l'été avait lentement accompli le voluptueux mariage du soleil de l'eau et de la terre dissocié de nouveau maintenant en principes élémentaires dans l'appareil compliqué et poussiéreux avec ses serpentins couverts de tartre calcaire ses cuves ses éléments de cuivre jaune piqué de taches vert de gris si serrées par endroits (il y avait, vers le haut, une sorte de couvercle vissé au pourtour dentelé d'entailles comme la tranche d'une pièce de monnaie et dans les rainures de laquelle s'était accumulée comme une moisissure d'un vert pâle vénéneux et presque blanc).

Parfois lorsqu'il sortait pour aller jusqu'à la vigne (coiffé alors de ce vieux panama jauni à ruban noir son éternel costume d'alpaga informe et décoloré flottant sur sa longue carcasse les deux tendons de son cou de chaque côté de la pomme d'Adam (et sur lesquels la peau ridée et hâlée semblait aussi flotter comme une enveloppe trop 
vaste) s'enfonçant sous le pied de col de sa chemise simplement fermée par un énorme bouton à tête de cuivre, et son éternelle et mince canne d'ébène à la main), ou à la cave assister au déchargement d'une charrette qui venait de passer dans l'allée annoncée d'abord par le grésillement du gravier sous les roues puis le bruit des chaînes de l'attelage les cris du charretier le tout croissant s'enflant se confondant dans un vacarme unique pierreux métallique et pour ainsi dire soufflant et suant tandis qu'elle passait devant la fenêtre et qu'au plafond du bureau mais en sens inverse tournaient comme les rayons d'une roue lumineuse dont le moyeu ou plutôt l'axe aurait été la mince fente entre les volets clos (puis le bruit décroissant tandis qu'elle s'engageait dans la cour et on ne savait quoi alors, une imperceptible modification de ce qui pénétrait de la lumière extérieure comme diffuse ou plutôt diffractée mollement éparpillée par l'opaque et blanc nuage de poussière soulevée restant suspendu dans l'air torride de septembre au-dessus de l'allée comme un sillage persistant et long à retomber), parfois donc il me confiait le soin de surveiller la pesée et je restais là dans cette intemporelle lumière d'un jaune foncé accroupi sur ma chaise les pieds sur le plus haut barreau les genoux relevés le menton soutenu par mes poings le visage à peu près à la hauteur du plateau de la table contemplant avec une sorte de fascination les gouttes se former lentement à l'extrémité du tube de caoutchouc dans l'imperceptible et minuscule trépidation qui se propageait à partir de la bouilloire semblable au bruit même du silence du temps comme un bouillonnement lointain paisible et sépulcral se confondant avec le bourdonnement monotone des insectes qui me parvenait à travers le grillage mangé de rouille, stagnant étale dans l'incandescente lumière de l'été finissant tandis que goutte après goutte je regardais monter imperceptiblement (ou plutôt tressaillir à intervalles réguliers sans qu'il fût possible à proprement dire de le voir s'élever pas plus que l'on ne voit avancer les aiguilles d'une montre) dans le col étroit de l'éprouvette le ménisque concave qui peu à peu approchait du repère.

Mais le plus souvent soit qu'il sortit sans m'avoir mis auparavant de garde devant l'appareil soit qu'il s'absorbât dans la rédaction de son courrier il oubliait de retirer à temps l'éprouvette d'où le liquide débordait ou bien se trompait ensuite dans ses mesures perdait le 
bout de papier (revers d'enveloppe, bande de journal) où il avait inscrit ses chiffres et tout était à recommencer ce qu'il entreprenait du reste sans jamais un mouvement d'humeur ou de colère au point que je soupçonnais même sa distraction ou sa négligence de faire partie de cet ensemble de précautions pour ainsi dire dont il s'entourait comme pour se protéger se ménager en quelque sorte des alibis contre l'inaction ou l'ennui avec cette sagacité roublarde de terrien en lutte contre les éléments dont il ne peut triompher que par chance ou ruse, s'efforçant donc ainsi de vaincre (ou de s'accommoder de ou de se concilier ou de signer un armistice avec) le temps, l'une de ses armes de ses secrets étant sans doute cette persévérante distraction ou étourderie qui lui permettait de justifier en quelque sorte la répétition des mêmes gestes se levant allant vider l'éprouvette inutile dans le petit évier aménagé dans un coin revenant sans hâte voûté semblant flotter dans cet informe costume d'alpaga rinçant la bouilloire se baissant pour examiner l'une après l'autre les bouteilles alignées le long de la plinthe marron gluantes de moût avec leurs étiquettes collées à la diable portant au crayon le numéro de la cuve et sur lesquelles pendaient comme une frange une herse irrégulière de bavures veineuses d'un pourpre foncé et enfin la nouvelle pesée mise en train de nouveau assis dans ces ténèbres diurnes à côté de l'appareil avec sa lampe veilleuse léchant de nouveau sans bruit les cuivres ternis comme la flamme de quelque culte secret géorgique et perpétuel célébré loin des regards dans la crypte d'un temple abandonné.

\section{III}

Regardant ces deux hommes au-dessous de moi en gare de Narbonne (moi dans le couloir du wagon) le vent nocturne déjà froid et noir de la fin septembre balayant le quai désert faisant osciller les lampes les cercles de lumière jaunâtre allant et venant sur le ciment gris les ombres des deux hommes oscillant aussi comme de fragiles télescopiques et grotesques doubles s'allongeant et se raccourcissant se rétractant puis s'étirant de nouveau tandis qu'ils s'affairent (pourquoi ? séparation, chacun d'eux allant à partir de maintenant dans une direction différente? se répartissant alors ce qu'ils avaient 
mis en commun ou mélangé au cours du voyage qui les a menés jusqu'ici ? - ou peut-être, le voyage fini et en prévision d'une marche à faire avec leurs bagages, équilibrant les charges ?) autour de leurs valises ouvertes l'une - en bois - posée sur le sol les deux autres en toile aux coins renforcés de pièces de cuir (ou plutôt sans doute un ersat 2 de cuir car déjà leurs bords et les éraflures présentent cet aspect gris et pelucheux du carton) sur un banc, le couvercle de la valise de bois blanc étant ouvert incliné en arrière exactement face au wagon de sorte que l'on peut voir collé à l'intérieur mais dans le sens de la longueur c'est-à-dire horizontalement une grande photo (sans doute la première page d'un illustré) de vedette de cinéma très jeune blonde aux gros seins la coiffure croulant asymétrique sur un côté du visage, et dans l'autre sens (c'est-à-dire perpendiculairement à la photo de la pin-up et dans l'intervalle entre un des côtés de celle-ci et le bord du couvercle) cinq ou six images pieuses format carte postale et en couleur représentant : la Vierge Marie la tête couverte d'un voile bleu pâle, un ciboire dont sort à demi une hostie entourée de rayons dorés, le Christ aux cheveux bouclés et à la barbe châtain clair écartant de ses mains les pans d'un manteau rouge-rose et montrant du doigt sur sa robe de lin un cœur sanglant lui aussi entouré de rayons, et encore deux scènes à plusieurs personnages impossible à bien distinguer du haut du wagon (une Nativité ? une Crucifixion ?) dans la lumière avare des lampes de la gare que le vent continue à balancer sans trêve.

Les deux hommes de ce type méditerranéen, sombre, taciturne patient et famélique (trace ou plutôt semence ou plutôt brune pollution ou plutôt éjaculation répandue par des générations de conquérants ou pirates arabes tout le long des côtes), le plus jeune des deux au visage basané aux cheveux noirs et ondulés la lèvre supérieure ornée d'une petite moustache, vêtu d'une chemise blanche, sans cravate, d'une veste gris clair, d'un pantalon plus foncé pendant flasque et mou avec des poches aux articulations, et dans l'échancrure de la chemise on peut voir le haut d'un tricot de corps, le tout complété par d'élégants souliers de cuir jaune tout fendillé ; l'autre plus âgé la nuque craquelée comme de la terre cuite couleur brique les cheveux gris et durs coupés en brosse le visage légèrement prognathe tout le corps petit et râblé portant une chemise à carreaux bleus et violets et un complet bleu à raies, costume qui a dû être dans le temps celui 
des dimanches parce que les pauvres ne peuvent jamais s'acheter lorsqu'ils ont un peu d'argent que des vêtements de fête qu'ils sont condamnés ensuite à porter sans fin comme de dérisoires témoins d'impossibles ambitions. Celui-là est chaussé de souliers de toile.

S'interrompent un instant pour allumer des cigarettes qu'ils tiennent ensuite entre le pouce et l'index, le bout allumé caché dans le creux de la main pour le protéger du vent.

Le plus âgé renroulant paisiblement l'amadou du briquet.

Le vent agitant (faisant frissonner) leurs cheveux comme sur la tête des morts (maures), gonflant (le vent) et faisant palpiter les multiples petits sacs de toile à carreaux (rouge et blanc, vert et blanc, violet et blanc) qui sont rangés à l'intérieur des valises à côtés d'effets et de linge plié.

Le plus jeune referme enfin le couvercle de la valise en bois sur lequel on peut voir alors calligraphié en pyrogravure (c'est-à-dire en brun et légèrement en creux) le nom :

\section{Jesus Nicolas Hernandez}

et ensuite ficelant longuement sur le couvercle une couverture d'un blanc sale pliée en huit.

Les valises refermées la couverture ficelée les dernières affaires rangées ils restent debout l'un à côté de l'autre sans se parler tirant silencieusement et économiquement sur leurs cigarettes, attendant sans doute le départ du train pour passer sur un autre quai ou peutêtre une correspondance qui doit venir plus tard, avec toujours à côté d'eux ce type à figure de rat ou d'oiseau (intendant? ou régisseur - ou propriétaire lui-même mais plutôt, à son allure, à son maintien, l'air d'un subalterne à la fois servile et hautain minable et méprisant : comptable, trésorier ou contremaître) venu les attendre et qui, coiffé d'un béret basque les deux mains dans les poches d'une gabardine cachou les a regardés sans intervenir pendant qu'ils refaisaient leurs valises se bornant à leur adresser de temps en temps quelques mots (conseils, ordres ?), frileux renfrogné ou plus simplement ennuyé.

Sur le banc de lattes peint en vert ils ont posé l'une des valises et une de ces gourdes en cuir pelucheux brun ou plutôt sans couleur, presque plate. 